\title{
Investigating the Integration of two Formal Methods
}

\author{
Juan Bicarregui ${ }^{1}$, Brian Matthews ${ }^{1}$, Brian Ritchie ${ }^{1}$ \\ and Sten Agerholm ${ }^{2}$ \\ ${ }^{1}$ Rutherford Appleton Laboratory Chilton, Didcot, Oxon, UK; ${ }^{2}$ IFAD, Odense M, Denmark
}

Keywords: B; Formal specification; Heterogeneous development; Translation; Tool support; VDM

Abstract. VDM and B are two mature formal methods currently in use by industry and supported by commercial tools. Though the methods are foundationally similar, the coverage of their supporting tools differ significantly. The SPECTRUM project has investigated the feasibility of integrating support for the two methodologies. In this paper, we describe the project and report on some technical results.

\section{Background}

VDM [Jon90] and B [Abr96] are two formal methods currently in use by industry and supported by mature commercial tools. The methods are essentially similar. Both are model-oriented methods for the development of sequential systems based on first order predicate calculus and set theory. Both have a set of proof rules defined for formal verification and validation. Both have a formal semantics: for $\mathrm{B}$ this is defined in terms of weakest preconditions, for VDM it is denotational.

However, the coverage of the supporting tools for VDM [ELL94] and B [BCORE] differ significantly in their capabilities for static analysis, proof, animation and code generation. This complementary functionality could benefit users from both communities, but because of the differences between the methods such cooperation is not currently possible. This paper reports progress towards the integration of these methods and tools within the EC project SPECTRUM ${ }^{1}$.

Correspondence and offprint requests to: Juan Bicarregui Rutherford Appleton Laboratory Chilton, Didcot, Oxon, OX11 0QX, UK. E-mail: jcb,bmm,br@inf.rl.ac.uk

1 EC ESPRIT project 23173, SPECTRUM, is a collaboration between: Rutherford Appleton Laboratory, GEC Marconi Avionics, Dassault Electronique, Institute of Applied Computer Science (IFAD), B-Core UK Ltd, Space Software Italia and Commissariat à l'Energie Atomique. For information about this project contact Juan Bicarregui. 


\subsection{VDM}

VDM's origins lie in the definition of programming language semantics in the 1970 s, but for many years it has been used in systems specification and development generally, and there is now an ISO standard [ISO96] of the specification language VDM-SL. It has a rich set of data type constructors, augmented by invariant predicates. Functions and state-transforming operations can be defined explicitly using a large expression and statement language or implicitly in terms of precondition and postcondition predicates.

The VDM-SL standard includes a denotational semantics [LaP95]. In particular, the strong type system supports static detection of many well-formedness errors. A published proof theory, described in [Jon90] and in greater detail in [BFL94], supports the validation of VDM-SL specifications through the discharge of proof obligations. The semantics is based on the three-valued Logic of Partial Functions (LPF [JoM94]) which explicitly deals with definedness of expressions and requires the demonstration of well-typing for the substitution of equals.

One area of weakness in VDM relative to B is its lack of generally agreed large-scale structuring. The standard contains a "informative annex" describing several alternative approaches to modules, including one implemented within the IFAD Toolbox and other structuring proposals exist[Fit91].

The IFAD VDM-SL Toolbox [ELL94] is an industrial strength commercially available tool which supports the ISO VDM-SL notation. The Toolbox includes a syntax checker, static semantic checker, and a pretty printer generating LaTeX output. In addition, it contains a debugger, an interpreter, and a $\mathrm{C}++$ code generator. It is also possible to perform test coverage analysis of specifications.

Other tools produced for VDM have included: Mural[JJL91] concentrating on proof obligation generation and discharge; SpecBox [SPEC] providing a parser, typechecker, semantic analyser and pretty-printer; the VDM Analysis Tool [DiF93] which provides test case generation; and VDM Through Pictures [DiL91] supporting the generation of VDM "skeletons" from EntityRelationship style diagrams.

\subsection{B}

The B-Method [Abr96] represents one of the most comprehensive formal methods currently being promoted as appropriate for commercial use. A development of VDM and Z, Jean-Raymond Abrial originated B whilst at the Programming Research Group at Oxford University in the early 1980s, and subsequently at British Petroleum Research (BP) and DIGILOG.

The method uses Abstract Machine Notation (AMN) which uses a notion of generalised substitution to represent state transformations, a style of specifying operations which is more "natural" to the programmer. The B-method also has powerful structuring mechanisms based on a notion of Abstract Machine which offers data encapsulation allowing modular design and development of systems.

B's underlying semantics is grounded in weakest preconditions over untyped set theory and classical logic; the type system is correspondingly weak, and the distinction between type-checking and proof is blurred.

The B-Toolkit [BCORE], developed by BP and subsequently by B-Core UK Ltd is an industrial strength commercially available tool to support formal de- 
velopment in B. It focuses on rigorous/formal design by supporting refinement from abstract specification through to imperative code. Tools exist for supporting static analysis (type-checking), dynamic analysis (animation), design documentation, proof of refinement and code generation. Another commercial support system is Atelier-B [Ate], provided by Steria Mediterranée which allows similar functionality to the B-Core Toolkit.

\subsection{Combining the Two Methods}

The combination of VDM and B was first explored in the MaFMeth Project [BDW96, BDM97]. This demonstrated that developing systems using a combination of B and VDM was both possible and useful. This project developed part of a transaction management system using VDM for the initial design and analysis and then translating into B for development and code generation. It was found that VDM encouraged a more abstract initial design, allowing clarity of expression, while B allowed more of a programming style suitable for the later stages of development. The formal notations allowed the use of many types of formal analysis, including syntactic and static analysis, test case generation, animation, formal proof, and code generation. Design features and errors were discovered at each analysis emphasising that the more analyses which can be brought to bear on a design the better.

The use of the combination of the two methods did however have problems. Errors were introduced in the translation between notations which was unsupported. Despite this, results of the study were favourable in terms of the total number of errors in the development, the early detection of errors and the overall effort expended.

Earlier work [BiR93], however, had shown that the languages are not trivially interchangeable. As well as the differences in structuring mechanisms, there are significant differences between the languages in their use of invariants, frames and postconditions. Furthermore there are significant differences between the languages in their underlying semantics and proof theories. B is based on classical logic whereas VDM uses the LPF which explicitly handles partial functions. This leads to a different treatment of termination and definedness.

Whilst we recognise that the two notations have some differences in interpretation, we believe that the semantics are sufficiently similar for interoperability to be meaningful and useful. In this paper, we discuss the feasibility of integrating the two languages.

\section{The Spectrum Programme}

The two methods are thus complementary in their styles: VDM is well-suited for requirements capture and high-level design, and $\mathrm{B}$ for subsequent development and code-generation. This is reflected by the functionality for various lifecycle tasks offered by the existing toolkits (see table 1). Support is best provided in those areas where each method is strong.

These observations have motivated the SPECTRUM programme which seeks to exploit this complementary nature by allowing the use of both languages within a single development process, including the integrated use of both toolkits. The first phase of this programme was the SPECTRUM project, a feasibility 
Table 1. Table showing the complementary nature of the Toolkits.

\begin{tabular}{|c|c|c|}
\hline Task & IFAD VDM-SL Toolbox & B-Toolkit \\
\hline Requirements capture & $\times$ & $\times$ \\
\hline Visualisation & $x$ & $x$ \\
\hline Abstract Specification & $\sqrt{ }$ & $x$ \\
\hline Type checking & $\sqrt{ }$ & $\sim$ \\
\hline Prototype code generation & $\sqrt{y}$ & $\sim$ \\
\hline Test coverage & $\sqrt{V}$ & $\times$ \\
\hline Animation/Execution & $\sqrt{ }$ & $\sqrt{ }$ \\
\hline Modularity & $\sim$ & $\sqrt{ }$ \\
\hline Refinement & $\times$ & $\sqrt{ }$ \\
\hline Proof & $x$ & $\sqrt{V}$ \\
\hline Final Code generation & $x$ & $\sqrt{ }$ \\
\hline Design documentation & $\times$ & $\sqrt{ }$ \\
\hline Version Control/Config Mgmt & $\times$ & $\sim$ \\
\hline
\end{tabular}

$\sqrt{ }$ good support; $\sim$ some support; $\times$ no support.

study which brought together four industrial users of formal methods in various safety-critical domains, the commercial suppliers of the VDM-SL Toolbox and the B-Toolkit, and a research laboratory with a mission of technology transfer. This feasibility study had three objectives:

- to assess the cost-effectiveness for the user partners, of a development process employing an integration of the VDM and B formal technologies,

- to determine the technical feasibility of this integration; and

- to investigate the commercial potential for the tool suppliers of the integration of supported forms of VDM and B.

The first objective was addressed through the development of user scenarios exploring the utility of the various functions available from the two toolkits and the advantages of employing them in terms of development cost and product quality. The scenarios were in the application domains of avionics systems and terrestrial transport embedded control. They were also reviewed from the perspectives of satellite communication control and nuclear power plant control. This work is reported elsewhere [Dra97, ALR98].

To address the third objective, the project assessed the commercial case for the provision of required functionality. It explored the overall cost-benefits of introducing the proposed method and the commercial viability of the proposed tool integration.

The remainder of this paper considers the second objective, the technical feasibility of the proposed integration. In this section, we describe the approach adopted based on translation between notations and in Sections 3 and 4, we describe the translations from B to VDM and from VDM to B respectively. The final section draws some conclusions.

\subsection{The SPECTRUM Lifecycle}

Figure 1 outlines the coverage of the VDM and B methods in terms of the classic V-lifecycle. VDM is seen as appropriate for high-level specification and analysis in the early stages of the development; B is more appropriate for lowlevel design and implementation. This approach must be supported by a facility 


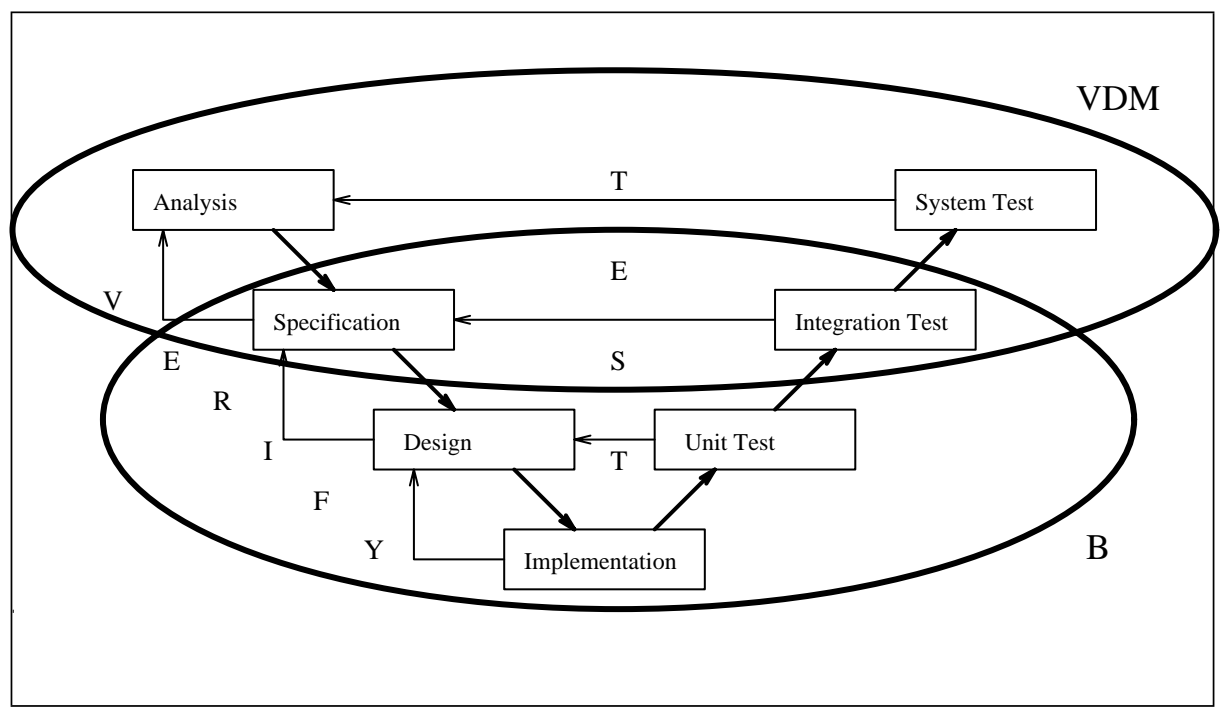

Fig. 1. The Lifecycle identified in the SPECTRUM project.

for moving from a VDM-SL model to a B model, which can be used as a starting point for refinement towards a B implementation machine from which code can be generated. At each stage of the development, verification with respect to the previous level is performed, and during implementation phases, testing is performed with respect to the appropriate level of the formal design.

As a consequence, in this lifecycle, the integration of VDM and $\mathrm{B}$ is required in two circumstances: translation from VDM-SL to B within the design process, and translations from $\mathrm{B}$ to VDM-SL within the testing phase.

\subsection{Integration through Translation}

We have seen that while the VDM-SL and B-AMN notations have broadly similar constructs and address similar problem areas, the two notations place different emphases on state structure, and on type and value expressivity. VDM provides a rich type definition language, and a similarly rich language of expressions and functions, but (relative to AMN) its state model is monolithic: in effect, the state model of a VDM-SL specification is a single variable of a complex type, and operations act on the state as a whole. On the other hand, whilst AMN provides a rich language for state modularisation, its type and expression notations are impoverished relative to VDM-SL. The state of a B specification consists of a relatively large number of state variables, usually of simple types, spread across a number of machines, each of which has operations acting primarily on the local state.

Thus, in translating between VDM-SL and AMN, two problems must be addressed: firstly how to represent the types and expressions of each language in the other, and secondly, how to relate the different complexities of structuring of the two languages. We first consider the translation of B-AMN to VDM-SL, and then VDM-SL to B-AMN. 


\section{Translating from B-AMN to VDM-SL}

The main difficulty of the translation integration of B and VDM is in translating the structuring mechanisms of B-AMN to VDM-SL. Its type, expression and substitution language is, to within first approximation, a subset of VDM's and can be translated directly ${ }^{2}$. We therefore focus on the translation of structuring.

The general approach is to translate each machine, except the top level machine, to a record with a field for each state variable. The top-level machine is translated to the VDM state with components for the included machines as well as for variables.

There are several structuring mechanisms in B which allow different degrees of hiding. We consider each of these in turn.

The Includes Clause. Machine inclusion is the major structuring mechanism for machines with state. It utilises a semi-hiding principle which allows the state of the included machine to be visible to the including machine, but only modified via its own operations. Each machine can only be included once in any other. This means that there can only exist one copy of a machine state in a hierarchy. For example, if a machine $C$ includes a machine $B$ and $B$ includes a machine $A$ then $C$ cannot also include $A$ explicitly, since $A$ is included via $B$, and from $C$ the state of $A$ can only be accessed via the operations of $B$. (On the other hand, the same machine may be "seen" or "used" multiple times; see later.)

We can model the includes clause by using an extra field in the VDM record types. The state of the included machine becomes a field in the state of the including machine. Consider for example three machines $X, Y$ and $Z$. Assume $X$ includes $Y$ and $Y$ includes $Z$, while $Z$ does not include other machines. The state of the three machines are represented using records. The "basic" machine $Z$ is represented by:

$$
\begin{array}{r}
Z:: z 1: Z 1 \\
z 2: Z 2
\end{array}
$$

$Y$ inherits $Z$ so it must have access to $Z$ state variables, e.g. in its invariant. Therefore $Z$ is an extra field in $Y$ 's record representation:

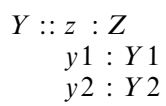

$X$ inherits $Y$ so we get:

$$
\begin{aligned}
X:: y & : Y \\
& x 1: X 1 \\
& x 2: X 2
\end{aligned}
$$

$X$ now has access to $Z$ via $Y$, so the transitivity is captured.

Next, consider B operations associated with machines. We represent these as functions in VDM-SL with extra arguments and results for before and after states. For example an operation of the machine $Z$ is parameterised with the record type $Z$ e.g.

$$
\text { opZ: } \operatorname{args} * Z \rightarrow \operatorname{res} * Z \text {. }
$$

\footnotetext{
2 A notable exception is B's parallel substitution which updates two variables simultaneously. However, this can be modelled in VDM using a let statement to calculate the two new values before doing the updates.
} 
An operation from machine $X, o p X$, may call an operation in $Y$ which is represented as follows (the $\mu$ operator modifies the named field of the given record type value with the given value):

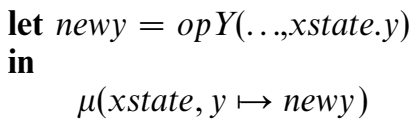

In turn op $Y$ may manipulate the state variables of $Z$ via a call to $o p Z$. However, this does not influence the translation of $o p X$.

The Sees Clause. The sees clause is more restricted than the includes clause. If a machine $X$ sees a machine $Y$ then $X$ can read the variables of $Y$ in its operations, but not in its invariant. It cannot write the variables and cannot call operations of $Y$. This means that $Y$ does not need to be an extra field of the machine record for $X$. It is enough to model it as an extra argument of operations. Thus we get:

$$
\text { opX: } \operatorname{args} * Y \rightarrow \text { res }
$$

The Uses Clause. The uses clause is similar to the sees clause and yet very different on one essential point: variables of the used machine can be referred to in the invariant of the using machine. The used variables must therefore be included in the record type modelling the using machine, as in the representation of the includes clause. However, unlike the includes clause, the uses clause can be used in multiple machines and this introduces a problem of multiple copies in the VDM translation. For example, suppose that two machines $Y 1$ and $Y 2$ each use a machine $Z$. Then if a machine $X$ is to include $Y 1$, it must also include not only $Z$, but also all other machines that use it (in this case $Y 2$ ). Consequently, the VDM record type for $X$ will contain three copies of the record for $Z$ (once directly, and twice indirectly via the records for $Y 1$ and $Y 2$ ). However, the $\mathrm{B}$ specification contains only one copy of $Z$. This can be resolved by specifying that these copies in the VDM record for $X$ must always be identical, for example by adding equality clauses to the invariant of the including machine of the form

$$
\text { zstate }=y . z \text { state } \text {. }
$$

However, since using machines cannot change the used state, we do not need to worry about preserving this part of the invariant in the VDM operations.

The used state variables are visible in operations of the using machine, but as mentioned above an operation of a using machine cannot change the state of the used machine. Moreover, used operations are not visible in using operations. Hence, in this respect the uses clause behaves like the sees clause and we could therefore copy the approach to translating the sees clause and extend the arguments of the using operations with an extra argument corresponding to the state of the used machine. However, this state is already available in the record of the using machine, so an extra argument is not necessary.

Generally we do not need to ensure that B restrictions on for example visibility with respect to the correct use of variables are satisfied in the VDM model. For example, although semi-hiding is lost in the translation, this loss is unimportant since B's restrictions on visibility will already have been checked within B and will be maintained by the translation.

Sets and Constants. Other components of machines are sets and constants. In $\mathrm{B}$, these are defined in properties clauses and may be non-deterministic. Sets are 
translated to both a new type and a value definitions, corresponding to the type of the set and the set of all elements of that type. Constants are represented as value definitions.

Guidelines for Translation. We can now present general guidelines for the translation of B into VDM. A B model is translated by working bottom-up, from basic machines with no "imports" towards the top level machine. State variables of non-top level machines become fields of a record type which has the same name as the machine. Trivial type machines such as Bool_TYPE just become types in VDM-SL, e.g. bool. Invariants of machines are translated to invariants of the record types. Initialisation is modelled using functions which call "init" functions of included machines.

Machine operations are represented as functions, where the machine state record is an extra argument and result. Parallel substitution in operations is handled using $\mu$-expressions, which are used to update fields of a record in parallel.

\begin{tabular}{|c|c|}
\hline B & VDM \\
\hline MACHINE $X$ & state $X$ of \\
\hline INCLUDES ${ }^{\lambda}$ & $y: Y$ \\
\hline VARIABLES & $x 1: X 1$ \\
\hline$x 1, x 2$ & $x 2: X 2$ \\
\hline $\begin{array}{l}\text { INVARIANT } \\
\quad x 1 \in X 1, x 2 \in X 2\end{array}$ & end \\
\hline $\begin{array}{c}\text { OPERATIONS } \\
\text { op } X=\ldots\end{array}$ & types \\
\hline END & $Y:: y 1: Y 1$ \\
\hline $\begin{array}{ll}\text { MACHINE } & Y \\
\text { SEES } Z & \end{array}$ & $y 2: Y 2$ \\
\hline $\begin{array}{l}\text { VARIABLES } \\
\quad \begin{array}{l}y 1, y 2 \\
\text { INVARIANT } \\
y 1 \in Y 1, y 2 \in Y 2\end{array}\end{array}$ & $\begin{array}{r}Z:: z 1: Z 1 \\
z 2: Z 2\end{array}$ \\
\hline $\begin{array}{l}\text { OPERATIONS } \\
\text { op } Y=\ldots\end{array}$ & operations \\
\hline END & $o p X() \ldots$ \\
\hline $\begin{array}{l}\text { MACHINE } \\
\text { VARIABLES } \\
z 1, z 2\end{array}$ & functions \\
\hline $\begin{array}{l}\text { INVARIANT } \\
\quad z 1 \in Z 1, z 2 \in Z 2 \\
\text { END }\end{array}$ & $\begin{array}{c}\text { op } Y(\text { yin }: Y, \operatorname{zin}: Z) \\
\text { yout }: Y \triangleq \\
\ldots\end{array}$ \\
\hline
\end{tabular}

Fig. 2. Summary of the translation from B to VDM.

For translating the top-level machine we use a VDM-SL state with an invariant and an initialisation predicate. Promoted operations become operations associated with the state by defining their body to call the lower-level operation.

Expressions in predicates are easy to translate since B's expression language is roughly speaking a subset of VDM's.

The translation is summarised in Fig. 2 which gives the translation of a representative partial specification in $\mathrm{B}$ and the equivalent translation in VDM. 
In this figure, machines $Y$ and $Z$ are translated as record types. The operation op $Y$ is translated as a VDM function taking type $Y$ as argument and result. Since machine $Y$ sees $Z$, the record type $Z$ is added as an argument to op $Y$. The top-level machine $X$ is used to define the VDM state model; this contains the (translations of the) variables of $X$ together with a value of type $Y$ (since machine $X$ includes $Y$ ). The operation $o p X$ of machine $X$ becomes an operation in the VDM specification.

\section{Translating from VDM-SL to B-AMN}

In translating from VDM-SL to B-AMN we have to consider, firstly, how to represent complex types and expressions within an impoverished expression syntax, and secondly, how to infer a structured state model from an unstructured model. This latter task requires the synthesis of a modular structure for the B specification from the type structure of the flat VDM specification. Further details on this synthesis are given in [MRB98].

Types and Expressions. It is possible to define a "reasonably literal" translation from VDM-SL to AMN, wherein type definitions, expressions and functions are translated as property-oriented (algebraic) definitions, and the (monolithic) toplevel VDM-SL state definition and operations are translated to a single AMN machine.

To explain this approach, consider the state of a representative VDM specification:

$$
\begin{gathered}
\text { state } S \text { of } \\
n: \mathbb{N} \\
a: A \\
\text { end }
\end{gathered}
$$

where $A$ is a user defined record type:

$$
\begin{array}{r}
A:: a 1: A 1 \\
a 2: A 2
\end{array}
$$

In the property oriented approach, since the record type $A$ is translated as an algebraic specification, it is given as the following stateless machine (truncated for brevity):

\section{MACHINE A_Type}

\section{SETS $A$}

\section{CONSTANTS}

$m k \_A, i n v \_A, a 1, a 2$

\section{PROPERTIES}

$$
\begin{aligned}
& a 1: A \rightarrow A 1 \wedge \\
& a 2: A \rightarrow A 2 \wedge \\
& m k \_A: A 1 \times A 2 \rightarrow A \wedge \\
& i n v \_A: A \rightarrow B O O L \wedge \\
& (\forall x x, y y) .(x x \in A 1 \wedge y y \in A 1 \Rightarrow \\
& \left.\quad\left(a 1\left(m k_{-} A(x x, y y)\right)=x x\right)\right)
\end{aligned}
$$


And a top level state machine of the form:

MACHINE $S$

SEES A_Type

VARIABLES $n, a$

INVARIANT

END

$n \in \mathbb{N} \wedge a \in A$

This is a literal translation of the VDM, and takes a similar approach to the translation of $\mathrm{Z}$ types into $\mathrm{B}$ proposed in [RBH94]. The algebraic VDM record type $A$ is translated into a stateless "property-oriented" machine, which declares the type as a set, a new $m k_{-} A$ constant as the constructor function, the fields as projection functions, and a new $i n v_{-} A$ constant as the invariant function. The behaviour of these constants is defined using properties, in effect giving an algebraic specification of the type. The properties clause soon becomes very large, with complex first-order logic and set theory expressions. Because of the relative weakness of the expression language in B to that in VDM-SL, these become hard to read, and the support tools for B find such expressions hard to deal with.

However, there are reasonably obvious translations from the VDM expression syntax to AMN's generalised substitutions. Though the latter are state transformers and not functional expressions, this suggests a translation approach that wherever possible "re-interprets" VDM functions as AMN operations that do not change the state.

Using the property oriented approach leads to a different "granularity" of the state than would be natural in a B specification. In normal usage, because of its flat state, VDM-SL uses record types for structuring data. On the other hand, because of its emphasis on generalised substitutions and primitive data types, AMN uses machines for structuring data.

In this paper We propose an approach to translation from VDM to B which attempts to cater for the differences in "state granularity" between VDM-SL and AMN. This translation synthesises a finer state structure from the VDM-SL record structure, use]ing the most appropriate style of $\mathrm{B}$ specification for the various parts of the VDM specification, resulting in a specification with a more distributed state.

\subsection{Preprocessing the VDM Specification}

First, we analyse the state model of the VDM-SL specification in a top-down manner to determine which type definitions contribute to the state. The manner in which a type is used is significant: if the state contains only individual values of a type (however deeply-nested in the type hierarchy), then we say that this type usage is simple and translate to machines with state variables for each field in the type and whose operations behave similarly to VDM record functions. However, if the state contains sets, sequences or maps of values of a type, then we say that the usage is complex and translate using "object-manager" machines, in a style similar to that given in [Lan96]. Here the creation and use of multiple (state) instances of the record type are controlled by a manager machine for that type. Other types, such as record types which appear as (non-state) arguments or results of functions or operations, are handled as property-oriented definitions. 
In this top-down analysis, the VDM spec is preprocessed to decide how to best translate each record type. Two sets are declared, Simple and Manager which represent those types which should be represented as a simple object machine, or as an object manager machine respectively. The analysis is a simple recursive procedure. First find the state, for example:

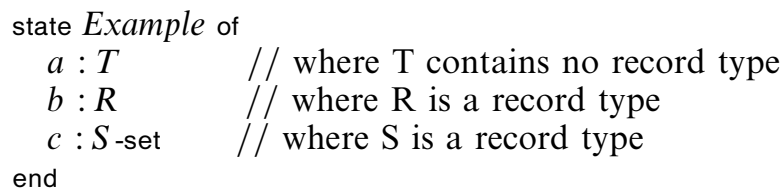

$T$ is any type which does not contain a record type, and it can be basic, user defined or an aggregate type (set, map or sequence) which do not have record types as members.

Any record types which are referenced directly, such as $R$, are added to the set Simple, and any record which is part of an aggregate, such as $S$, are added to the set Manager.

This process is repeated for each record type $R \in$ Simple, and $S \in$ Manager, with the additional conditions:

1. if $S \in$ Manager, then $S \notin$ Simple;

2. if record $R$ occurs in a field of $S \in$ Manager, then $R \in$ Manager.

Thus if a record type $S$ is added to Manager, it must be removed from Simple, and any records referenced from $S$ must also be added to Manager.

\subsection{Inferring a Structured Model}

When the preprocessing is complete, then machines can be generated. All record types in Simple are translated as simple structured object machines, while types which are in Manager are translated as object manager machines. Any record types not in either of these sets are translated as property oriented stateless machines.

Thus there are three cases to consider of records to translate: a Simple record with a reference to a Simple record within it; a Simple record with a reference to a Manager record; and a Manager record with a reference to a Manager record. The final case of Simple within Manager will not occur.

In the first case, if for example, $\mathrm{P} \in$ Simple such that:

$$
\begin{aligned}
P:: p 1: T & / / \text { non record type } \\
p 2: R & / / \text { record type such that } \mathrm{R} \in \text { Simple }
\end{aligned}
$$

then we would generate the simple state machine:

MACHINE $\quad P_{-} O b j$

INCLUDE $p 2 . R_{-} O b j$

VARIABLES

p1

\section{INVARIANT}

$p 1 \in T$

END 
Note that the machine $R_{-} O b j$ is included into this machine with a renaming, using the convenient name $p 2$. This machine may well be used elsewhere in the specification, and each including machine needs a unique copy.

$R_{-} \mathrm{Obj}$ is a simple state machine derived as follows. If:

$$
\begin{aligned}
R:: r 1 & : R 1 \\
r 2 & : R 2
\end{aligned}
$$

In the translation, the record $R$ is split into its fields, and give a state variable defined for each:

MACHINE R_Obj

VARIABLES

$r 1, r 2$

\section{INVARIANT}

$r 1 \in R 1 \wedge r 2 \in R 2$

END

The higher-level state machine $P_{-} O b j$ which includes this represents the "inheritance" of the datatype:

If the data types $R 1, R 2$ are themselves Simple or Manager record types they can be broken down further into similar machines. Thus we build a hierarchy of machines which preserve the structure of the VDM specification, but have a finer state granularity. This specification is much clearer and easy to work with, exploiting as it does the strength of B and B tools in manipulating machine state and generalised substitutions.

For the second case, if $\mathrm{P} \in$ Simple such that:

$$
\begin{aligned}
P:: p 1: T & / / \text { non record type } \\
p 2: S & / / \text { record type such that } \mathrm{S} \in \text { Manager }
\end{aligned}
$$

then generate the simple state machine:

MACHINE P_Obj

INCLUDE $S \_M g r$

VARIABLES

$p 1, p 2$

INVARIANT

$p 1 \in T \wedge p 2 \in$ sids

END If

In this case $S \_M g r$ is an Pobject manager machine for the type $S$, as follows.

$$
\begin{array}{r}
S:: s 1: S 1 \\
s 2: S 2
\end{array}
$$

then we translate this as:

MACHINE $S \_M g r$

SETS $S_{-} I d s$ 


\section{VARIABLES}

sids, $s 1, s 2$

\section{INVARIANT}

$$
\begin{aligned}
& s i d s \subseteq S_{-} I d s \wedge \\
& s 1 \in \operatorname{sids} \rightarrow S 1 \wedge \\
& s 2 \in \operatorname{sid} s \rightarrow S 2
\end{aligned}
$$

END

Typically, an object manager machine will also include some basic operations for inspecting and manipulating the variables; for example equality should be defined on the value of the attributes rather than on the identifier. In this paper, these are omitted for clarity.

Thus using an object manager machine, aggregates can be formed and manipulated. Note that there is no renaming carried out here. There is only one object manager machine in the system, and all references should be to that machine. However, there is an issue here: the rules of composition in $\mathrm{B}$ allow a machine to be included in only one other machine. Thus if the manager record is referred to from more than one record type, this condition may be broken. The resolution of this problem would be to break down the $S \_M g r$ machine into two, with a simple machine declaring the abstract set $S_{-} I d s$, representing the object identifiers, and a manager machine. The manager machine is then included with renaming, and the set is accessed via the SEES construct. This allows the same set of object identifiers to be used across different object managers.

For the third case, if $\mathrm{P} \in$ Manager such that:

$$
\begin{aligned}
P:: r 1: T & / / \text { non record type } \\
r 2: S & / / \text { record type such that } \mathrm{S} \in \text { Manager }
\end{aligned}
$$

then generate the object manager machine as follows, which includes a further object manager machine $S \_M g r$.

MACHINE $P \_M g r$

INCLUDE $S \_M g r$

SETS $P_{-} I d$

VARIABLES

pids, $r 1, r 2$

\section{INVARIANT}

pids $\subseteq P_{-} I d \wedge$

$r 1 \in$ pids $\rightarrow T \wedge$

$r 2 \in$ pids $\rightarrow$ sids

\section{END}

Other types and record types, which are not accessed via the state model are treated by a property oriented translation.

It may also be necessary to give property oriented translations as well as state based ones for certain records; they are used as input/output to functions for example. The analysis of the specification can be extended to cover this eventuality. 
The translation presented so far concentrates on the different data models of VDM and B. To present a full translation, other aspects of the languages need to be considered, especially the type and expression language, and the operation and function language, which is beyond the topic of this paper.

\section{A Worked Example}

To illustrate the different approaches, we consider the following small example of a VDM-SL specification taken from a SPECTRUM user scenario concerning the management of the doors on metro trains.

This example was initially designed using OMT from which a VDM specification was derived. The fragment given here has a state Metro, with a train component. The train itself is a record with two components, motion, representing the status of the train's motion, and the current speed of the train. The invariant on the train states that when the train is stopped, its speed is zero. We also provide a function for braking.

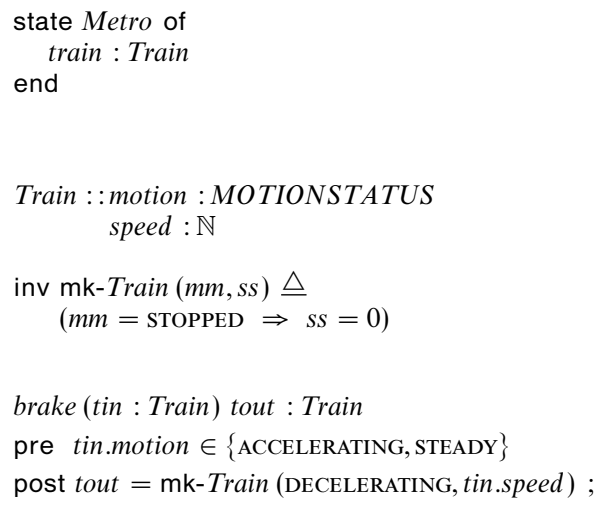

Under the property oriented translation, this becomes two machines, one with the state model:

MACHINE Metro_Type

SEES

Train_Type

VARIABLES

train

INVARIANT

train $\in$ Train

END

The other machine is a stateless property-oriented specification, with the invariant represented as a complex first-order logic formula in the properties clause, and the brake represented as a stateless operation:

MACHINE Train_Type

SETS Train0 


\section{CONSTANTS}

Train, mk_Train, inv_Train, motion, speed, init_Train

\section{PROPERTIES}

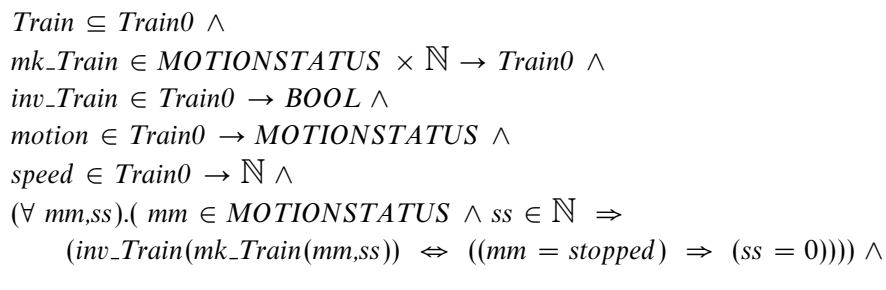

\section{OPERATIONS}

tout $\leftarrow$ brake $(\operatorname{tin}) \hat{=}$

PRE

tin $\in$ Train $\wedge$

motion $($ tin $) \in\{$ accelerating , steady $\}$

THEN

END

tout : = mk_Train ( decelerating , speed $(\mathrm{tin}))$

END

In this latter machine, functions are represented as stateless operations, which permits a more expressive syntax than translating them as properties. Nevertheless, this is an awkward machine to manipulate in the B-Method, and while perfectly valid in the language, is not a "natural" approach in B.

However, using the synthesis method, after an analysis which determines that the train record type is a Simple type, it is translated into a machine with state variables.

MACHINE Train_Obj

VARIABLES

motion, speed

\section{INVARIANT}

motion $\in$ MOTIONSTATUS ^

speed $\in \mathbb{N} \wedge$

$($ motion $=$ stopped $) \Rightarrow($ speed $=0)$

\section{OPERATIONS}

brake $\widehat{=}$

PRE

THEN

motion $\in\{$ accelerating , steady $\}$

END

motion : = decelerating

END

This machine is then "included" into the top-level Metro specification, with a renaming. Metro is otherwise the same as in the property oriented approach. This is a much more natural style in B using more state variables of simple types. This 
machine can be easily included into a continuing B development and resulting proof obligations more easily expressed and discharged.

In effect, the proposed translation automates the extraction of design information from the VDM-SL specification, by deriving a finer-grained state model. Design is an intelligent process, and inevitably this design information may not be exactly as the user intended; an element of user supervision may be required here to judge whether the automatic design is suitable. However, as this is automatically generated, this useful structuring can be gained at low cost.

A useful test was the application of the B-AMN to VDM-SL translation given earlier to this latter specification. It produced a VDM specification very similar to the original, supporting our claim that the translations given here have a high degree of symmetry.

The definition of the translation is not yet complete. Some work is still required to provide a complete account of translating VDM functions and operations, and VDM full type and expression language language needs to be tackled. Nevertheless, this approach seems to be promising, practical and useful.

\section{Conclusion}

A prerequisite of the benefits to be gained from heterogeneous development using VDM and $\mathrm{B}$ is a smooth integration of the methods which maintains the correctness of the development while adding little or no cost. The reported project demonstrated the feasibility of combining the two methodologies, and their supporting toolkits. In particular it explored the translations between the two notations. The translation of B-AMN to VDM-SL was shown to be a more straightforward task than the translation from VDM-SL to B-AMN, although ultimately, both are feasible.

The proposed VDM to B and B to VDM translations are largely symmetric. The translation from B-AMN to VDM-SL involves the loss of state structuring and its replacement with richer type structure using a bottom-up analysis of the specification. The translation from VDM-SL to B-AMN, which replaces mathematical expressiveness in the type system with design in the machine structure, reflects B's approach of being close to real machine types. The required machine structure can be largely inferred automatically by a top down analysis of the VDM and thus an automatic translator could really be seen as a adding value to the source specification.

The translation automates the extraction of design information from the VDMSL specification, by deriving a finer-grained state model. As the design process requires intelligent insight, the resultant design may not be in the form the user desires, and therefore an element of user judgement is still required to determine which elements of this design are appropriate. However, as the design elements have been automatically generated, the useful elements have been gained at low cost.

Thus the SPECTRUM project has advanced the understanding of the technical issues involved in the integration of VDM and B and the approach enabled by these translations yields the possibility of a formal development offering the advantages of both VDM and B and their respective tools. Each method can then be used in the lifecycle at those points for which it is most appropriate.

This work is not yet complete and further research is needed before support for such translations can be developed. The translations have been derived through 
experience of the notations and have not been presented fully formally, nor have they been justified formally with respect the semantics of the two notations. VDM has a denotational semantics over a logic for partial functions and B has a weakest precondition semantics over classical logic. A formal proof of the preservation of semantics in the translations would require a mapping between the semantic models similar to that given in the weakest precondition semantics for $\mathrm{Z}$ in [CaW98]. However, in this case, the difference between the logics adds an extra concern. Recent developments in B, such as hypersubstitutions [DuS96] and "well-defined B" [BBM98] may aid semantic compatibility here. An investigation into the formal relationship between the methods is the subject of current research under the UK EPSRC funded project "VDM+B" which will investigate the semantic mapping necessary to underpin the translations presented here.

To date, the penetration of formal methods into mainstream software development has been extremely slight. One reason for this might be that the confusion amongst users concerning which methods are most appropriate for which applications does not encourage the take up of formal techniques in general. In bringing together the methods and tools for VDM and B the SPECTRUM project should also help to bring together their user communities and hence provide increasing evidence of the feasibility of the formal approach.

\section{Acknowledgements}

We would like to thank our partners on the SPECTRUM project and the Commission of the European Union for their support.

\section{References}

[Abr96] Abrial, J.-R.: The B-Book: Assigning Programs to Meaning, CUP, 1996.

[ALR98] Agerholm, S., Lecoeur, P.-J. and Reichert, E.: Formal Specification and Validation at Work: A Case Study using VDM-SL. Proceedings of Second Workshop on Formal Methods in Software Practice, Florida, ACM, March 1998.

[Ate] Atelier, B.: Steria Mediterranée, The B Page URL $<$ http://www.atelierb.societe.com/PAGE_B/uk/bhomepg.htm>

[BCORE] The B-Toolkit, B-Core (UK) Ltd. Welcome page URL <http://www.b-core.com>

[BBM98] Behm, P., Burdy, L. and Meynadier, J.-M.: Well-defined B. Proceedings of the 2nd B Conference, Ed. Bert, LNCS 1393, Springer-Verlag, 1998.

[BDW96] Bicarregui, J. C., Dick, A.J.J. and Woods, E.: Quantitative Analysis of an Application of Formal Methods. In: Proceedings of Formal Methods Europe'96, LNCS 1051, ed. M-C. Gaudel and J. Woodcock, Springer-Verlag 1996.

[BDM97] Bicarregui, J. C., Dick, A.J.J., Matthews, B.M. and Woods, E.: Making the Most of Formal Specification through Animation, Testing and Proof, Science of Computer Programming 29, pp. 53-78, 1997.

[BFL94] Bicarregui, J. C., Fitzgerald, J. S., Lindsay, P. A., Moore, R. and Ritchie, B.: Proof in VDM: a Practitioner's Guide. Springer-Verlag, 1994.

[BiR93] Bicarregui, J. C. and Ritchie, B.: Invariants, Frames and Preconditions: A Comparison of the VDM and B Notations. In: Proceedings of Formal Methods Europe'93, LNCS 670, ed. J. Woodcock and P G Larsen, Springer-Verlag, 1993.

[CaW98] Cavalcanti, A. and Woodcock, J.: A Weakest Precondition Semantics for Z. The Computer Journal, 41, No. 1, 1998.

[DiF93] Dick, A. J. J. and Faivre, A.: Automating the Generation and Sequencing of Test Cases from Model-based Specifications. In J.C.P. Woodcock and P.G. Larsen, editors, FME'93: Industrial-Strength Formal Methods, LNCS 670, Springer-Verlag, 1993.

[DiL91] Dick, A. J. J. and Loubersac, J.: A Visual Approach to VDM: Entity-Structure Diagrams, Technical Report DE/DRPA/91001, Bull, 68, Route de Versailles, 78430 Louveciennes (France), 1991. 
[Dra97] Draper, J.: (ed) Industrial benefits of the SPECTRUM approach SPECTRUM Project External Deliverable 1.3, 1997. See http://www.dci.clrc.ac.uk/Activity.asp?SPECTRUM.

[DuS96] Dunne, S. and Stoddart, W.: Hypersubstitutions: Extending Generalised Substitutions to Model Semi-decidable Operations. Proceedings of the 1st Conference on the B-Method, Nantes, France, Ed. H. Habrias, ISBN : 2-906082-25-2, 1996.

[ELL94] Elmstrøm, R., Larsen, P. G. and Lassen, P. B.: The IFAD VDM-SL Toolbox: A Practical Approach to Formal Specifications, ACM Sigplan Notices 29(9), pp. 77-80, 1994. For more information see http://www.ifad.dk/

[Fit91] Fitzgerald, J. S.: Modularity in Model-Oriented Specification and its Interaction with Formal Reasoning. Ph.D. Thesis, University of Manchester, 1991.

[ISO96] ISO Information Technology - Programming Languages - Vienna Development MethodSpecification Language. Part 1: Base Language. ISO 13817-1, 1996.

[Jon90] Jones, C. B.: Systematic Software Development Using VDM, 2nd Edition, Prentice-Hall, 1990.

[JoM94] Jones, C. B. and Middelburg, C. A.: A Typed Logic of Partial Functions Reconstructed Classically, Acta Informatica, 1994.

[JJL91] Jones, C. B., Jones, K. D., Lindsay, P. A. and Moore, R.: Mural: A Formal Development Support System. Springer-Verlag, 1991.

[Lan96] Lano, K.: The B Language and Method: a guide to practical formal development. SpringerVerlag 1996.

[LaP95] Larsen, P. G. and Pawlowski, W.: The Formal Semantics of ISO VDM-SL, Computer Standards and Interfaces, 17, numbers 5-6, 1995.

[MRB98] Matthews, B. M., Ritchie, B. and Bicarregui, J. C.: Synthesising Structure from Flat Specifications, Proceedings of the 2nd B Conference, Ed. Bert, LNCS 1393, SpringerVerlag, 1998.

[RBH94] Ritchie, B., Bicarregui, J. C. and Haughton, H.: Experiences in Using the Abstract Machine Notation in a GKS Case Study. Proceedings of Formal Methods Europe '94, Eds. Naftalin, Denvir, and Bertran, LNCS 873, Springer-Verlag, 1994.

[SPEC] Specbox. For further information contact: Peter Froome, Adelard, Coborn House, 3 Coborn Road, London E3 2DA, UK.

Received March 1998

Accepted in revised form October 1998 